\title{
Impact on Decision Making Framework for Medicine Appraisal in Chinese Public Hospital Decision- making: Determining the Value of Five DPP-4 inhibitors
}

\section{Yun Bao}

Gansu Provincial Hospital

Bei Gao

Gansu Provincial Hospital

Min Meng

Gansu Provincial Hospital

Bin Ge

Gansu Provincial Hospital

Yan Yang

Gansu Provincial Hospital

Chunchun Ding

Gansu Provincial Hospital

Bingyin Shi

The First Affiliated Hospital of Xi'an Jiaotong University

Limin Tian ( $\nabla$ tlm6666@sina.com )

Gansu provincial hospital https://orcid.org/0000-0002-4769-4375

\section{Research article}

Keywords: Evidence and Value, Impact on Decision Making(EVIDEM) framework, Multi-criteria decision analysis (MCDA), Hospital-based health technology assessment(HB-HTA)

Posted Date: July 28th, 2020

DOl: https://doi.org/10.21203/rs.3.rs-35171/v1

License: (c) (1) This work is licensed under a Creative Commons Attribution 4.0 International License. Read Full License

Version of Record: A version of this preprint was published at BMC Health Services Research on August 12th, 2021. See the published version at https://doi.org/10.1186/s12913-021-06827-0. 


\section{Abstract}

Background: Decision making for medicine optimization in Chinese public hospitals is decided by a hospital Pharmacy Management Committee (PMC) and is complex, subjective and requires efficient methods to ensure transparency and consistency for the factors being considered. This study aimed to evaluate the EVIDEM framework in medicine optimization for Chinese public hospitals. In this study DPP-4 inhibitors were appraised.

Methods: Following EVIDEM methodology, we convened an appraisal group and asked each individual to express their perspectives by assigning weights to each criteria. The estimated value of the five DPP-4 inhibitors was obtained by Multi-Criteria Decision Analysis (MCDA) which combined individual weighting (between 1 and 5) of each criterion with individual scoring for each intervention in each criterion.

Results: A systematic literature search was completed and the committee assigned weights for each criterion. The overall value orders for each DPP-4 inhibitor included Sitagliptin (0.45), Linagliptin (0.44), Vildagliptin (0.43), Alogliptin (0.42) and Saxagliptin (0.40).

Conclusions: This study was the first pilot using the EVIDEM framework and MCDA for medicine optimization in a Chinese public hospital. We aimed to improve the transparency and consistency of decision-making by using multi-criteria decision analysis while also discussing its limitations.

\section{Background}

The Pharmacist Management Committee (PMC) makes decisions regarding medicine optimization in Chinese public hospitals[1, 2]. This decision-making progress is complex, subjective and requires both efficient and explicit methods to ensure transparency and consistency of the participating factors. Administration literature, such as work focused on cost-effectiveness analysis, does not systematically consider the multi-dimension value of a drug $[3,4]$.

Multicriteria decision analysis (MCDA) is a method used to appraise alternatives for the individual that often have conflicting criteria by combining these into a single appraisal[5]. Currently, MCDA is applied to health care decision making such as hospital purchasing [6, 7]. MCDA helps increase the consistency, transparency and legitimacy of decision making[8]. The Evidence and Value: Impact on Decision Making (EVIDEM) collaboration developed an EVIDEM framework that includes a core model containing 13 criteria that are adaptable to a contextual tool[9] (Table 1,2). EVIDEM framework bridges the MCDA with health technology assessment (HTA)[10]. 
Table 1

EVIDEM Core Model

\begin{tabular}{|c|c|}
\hline Domains & Criteria \\
\hline \multirow[t]{3}{*}{ Need for Intervention } & Disease severity \\
\hline & Size of affected population \\
\hline & Unmet needs \\
\hline \multirow[t]{3}{*}{ Comparative Outcomes of Intervention } & Comparative effectiveness \\
\hline & Comparative safety/tolerability \\
\hline & Comparative patient-perceived health / PRO \\
\hline \multirow[t]{2}{*}{ Type of Benefit of Intervention } & Type of preventive benefit \\
\hline & Type of therapeutic benefit \\
\hline \multirow[t]{3}{*}{ Economic Consequences of Intervention } & Comparative cost consequences - cost of intervention \\
\hline & Comparative cost consequences - other medical costs \\
\hline & Comparative cost consequences - non-medical costs \\
\hline \multirow[t]{2}{*}{ Knowledge about Intervention } & Quality of evidence \\
\hline & Expert consensus/clinical practice guidelines \\
\hline
\end{tabular}

Table 2

EVIDEM Contextual tool

\begin{tabular}{|ll|}
\hline Domains & Criteria \\
\hline Normative Contextual Criteria & Mandate and scope of healthcare system \\
\cline { 2 - 2 } & $\begin{array}{l}\text { population priorities and access } \\
\text { Common goal and specific }\end{array}$ \\
\cline { 2 - 2 } & Environmental impact \\
\hline Feasibility Contextual Criteria & System capacity and appropriate use of intervention \\
\cline { 2 - 2 } & Political/historical/cultural context \\
\hline Opportunity Cost & Opportunity costs and affordability \\
\hline
\end{tabular}

DPP-4 inhibitors are anti-diabetic drugs that work by inhibiting the activation of the DPP-4 receptor. There are currently five DPP-4 inhibitors that are marketed in China including Sitagliptin which was approved in 2006, Linagliptin approved in 2011, Vildagliptin approved in 2007, Alogliptin approved in 2013 and Saxagliptin approved in 2009[11]. It is difficult to choose the most suitable medication when considering hospital purchasing while remaining fair, transparent and consistent.. 
The aim of this study was to appraise DPP-4 inhibitors with the goal of piloting the EVIDEM framework and MCDA to identify the most optimal medication.

\section{Methods}

Methodology of the EVIDEM framework was followed for MCDA[9]. MCDA was performed following reports published by ISPORT[12, 13]. The estimating program is shown in Fig. 1.

First, PubMed, Embase, The Cochrane Library and China Biology Medicine disc (CBM) from July 2019 onward were searched. Search terms included the five DPP-4 inhibitors and placebos, type 2 diabetes, clinical efficacy, safety, quality of life/QoL/HRQoL, epidemiol*/prevalence/incidence, mortality, guidelines, recommendations, clinical practice, patient-reported outcome*/PRO, cost*, econom*, meta-analysis and net meta-analysis. For the factors that are country-specific (e.g. cost-effectiveness and clinical guidelines), data was only included based on Chinese settings. Placebo was chosen as the comparator. To similarly compare the five DPP-4 inhibitors, meta-analysis comparing the five DPP-4 inhibitors with placebo was first performed. The HTA group analyzed and synthesized the data.

Second, a committee comprising two doctors, one nurse, one pharmacist, one health economist and one decision-maker was generated. All six members were already staff at the Gansu provinal hospital. Both the doctor and nurse worked in the Endocrinology Institute.

Next, following EVIDEM methodology, the committee assigned weight for each criterion according to their importance before it was known which medicines needed to be evaluated. Criterion scored as 5 were considered the most important and those scored as 1 were considered the least important.

Based on the evidence matrix synthesized by the HTA group (Appendix 1), experts assigned a score for each criterion. The scores for the absolute criteria (those that were not compared to placebo) ranged from 0 to 5 , where 0 was the lowest value and 5 was the highest. For relative criteria (those compared to the placebo), the scale ranged from - 5 to 5 to reflect the full range of comparative effects. Criteria in the contextual tool were discussed.

Finally, a value estimate $(\mathrm{V})$ of the intervention was calculated based on a linear additive model as a sum of the value contributions $\left(\mathrm{V}_{\chi}\right)$ [or combined normalized weights $\left(\mathrm{W}_{\chi}\right)$ and standardized scores $\left(\mathrm{S}_{\chi}\right)$ ] of all $(n)$ criteria of the quantitative EVIDEM Core Model. Each estimated value was transformed to a 0-1 scale.

\section{Results}

\section{Criterion Weights}

Members of the panel committee discussed and decided on a mean weight for every criterion as shown in Fig. 2. Every criterion was of high relevance besides the comparative cost consequences-non-medical cost $(2.87 \pm 1.03)$. The panelists decided the most important criterion to be the quality of evidence $(4.01 \pm 0.52)$. Interestingly, six of the criteria included disease severity, size of the affected population, comparative 
effectiveness, type of therapeutic/preventive benefit and cost of intervention, which were all assigned the same weight of 3.58. The type of therapeutic/preventive benefit and non-medical cost showed high variability of 1.17 and 1.07 , respectively.

2. Scores for the five DPP-4 inhibitors

Mean scores for the five DPP-4 inhibitors are presented in Table 3. Criteria including disease severity, size of the affected population, unmet need, type of therapeutic/preventive benefit, quality of evidence and expert consensus/clinical practice guidelines were evaluated using absolute terms with a score between 0 and 5. All of these criteria were given the same scores for all five medicines. According to the International Diabetes Federation report, the number of diabetic patients in China was 114 million in 2017 and more than $90 \%$ were diagnosed with type 2 diabetes. These numbers are expected to increase to 642 million by 2040[14]. Data from Gansu Province show that the prevalence of type 2 diabetes in 2014 was 9.7\%[15]. Based on this, the size of affected population showed the highest score and lowest variability $(4.33 \pm 0.52)$ in absolute criteria. Disease severity $(4.00 \pm 1.26)$ and type of therapeutic benefit $(4.00 \pm 0.00)$ followed affected population size. In contrast, the committee did not estimate the type of preventive benefit since there was no data available on this topic. Quality of evidence showed the lowest score (3.33 \pm 0.82$)$ based on three meta-analyses evaluated with high bias by the ROBIS tool[16]. Expert consensus/clinical practice guidelines showed the highest variability (SD 1.51). 
Table 3

Mean scores of criterions of five DPP- 4 inhibitors

\begin{tabular}{|c|c|c|c|c|c|c|c|c|c|c|}
\hline & \multicolumn{2}{|c|}{ Saxagliptin } & \multicolumn{2}{|c|}{ Alogliptin } & \multicolumn{2}{|c|}{ Sitagliptin } & \multicolumn{2}{|c|}{ linagliptin } & \multicolumn{2}{|c|}{ Vildagliptin } \\
\hline & Mean & SD & Mean & SD & Mean & SD & Mean & SD & Mean & SD \\
\hline Disease severity & 4.00 & 1.26 & 4.00 & 1.26 & 4.00 & 1.26 & 4.00 & 1.26 & 4.00 & 1.26 \\
\hline $\begin{array}{l}\text { Size of affected } \\
\text { population }\end{array}$ & 4.33 & 0.52 & 4.33 & 0.52 & 4.33 & 0.52 & 4.33 & 0.52 & 4.33 & 0.52 \\
\hline Unmet needs & 3.50 & 0.55 & 3.50 & 0.55 & 3.50 & 0.55 & 3.50 & 0.55 & 3.50 & 0.55 \\
\hline $\begin{array}{l}\text { Comparative } \\
\text { effectiveness }\end{array}$ & 3.50 & 1.64 & 4.33 & 1.03 & 4.67 & 0.52 & 4.33 & 1.03 & 3.67 & 1.51 \\
\hline $\begin{array}{l}\text { Comparative } \\
\text { safety/tolerability }\end{array}$ & 0.17 & 0.98 & 0.33 & 0.82 & 0.83 & 0.75 & 0.67 & 1.21 & 0.67 & 0.82 \\
\hline $\begin{array}{l}\text { Comparative } \\
\text { patient-perceived } \\
\text { health / PRO }\end{array}$ & -0.17 & 0.41 & -0.33 & 0.52 & -0.17 & 0.41 & -0.17 & 0.41 & -0.50 & 0.55 \\
\hline $\begin{array}{l}\text { Type of } \\
\text { therapeutic benefit }\end{array}$ & 4.00 & 0.00 & 4.00 & 0.00 & 4.00 & 0.00 & 4.00 & 0.00 & 4.00 & 0.00 \\
\hline $\begin{array}{l}\text { Type of preventive } \\
\text { benefit }\end{array}$ & / & / & / & / & / & / & / & / & / & / \\
\hline $\begin{array}{l}\text { Comparative cost } \\
\text { consequences - } \\
\text { cost of } \\
\text { intervention }\end{array}$ & -1.83 & 1.33 & -1.83 & 1.33 & -1.50 & 0.84 & -1.33 & 0.82 & -1.17 & 0.98 \\
\hline $\begin{array}{l}\text { Comparative cost } \\
\text { consequences - } \\
\text { other medical } \\
\text { costs }\end{array}$ & I & / & / & / & I & I & / & I & / & / \\
\hline $\begin{array}{l}\text { Comparative cost } \\
\text { consequences - } \\
\text { non-medical costs }\end{array}$ & -1.83 & 1.47 & -1.67 & 1.51 & -1.33 & 1.03 & -1.50 & 1.05 & -1.33 & 1.21 \\
\hline $\begin{array}{l}\text { Quality of } \\
\text { evidence }\end{array}$ & 3.33 & 0.82 & 3.33 & 0.82 & 3.33 & 0.82 & 3.33 & 0.82 & 3.33 & 0.82 \\
\hline $\begin{array}{l}\text { Expert } \\
\text { consensus/clinical } \\
\text { practice guidelines }\end{array}$ & 3.67 & 1.51 & 3.67 & 1.51 & 3.67 & 1.51 & 3.67 & 1.51 & 3.67 & 1.51 \\
\hline
\end{tabular}

Comparing the interventions to placebo, five DPP-4 inhibitors showed greater added value for effectiveness. Sitagliptin showed the highest score for effectiveness (4.67 \pm 0.52$)$. Alogliptin and Linagliptin both had the same scores $(4.33 \pm 1.03,4.33 \pm 1.03)$. Vildagliptin and Saxagliptin both had scores that were less $(3.67 \pm 1.51,3.50 \pm 1.64)$ than those of Alogliptin and Linagliptin. In contrast, medicine showed a lower score and higher variability when analyzing comparative safety. These scores 
were $0.83 \pm 0.75$ for Sitagliptin, $0.67 \pm 1.21$ for Linagliptin, $0.67 \pm 0.82$ for Vildagliptin, $0.33 \pm 0.82$ for Alogliptin and $0.17 \pm 0.98$ for Saxagliptin. Comparative patient-perceived outcomes/PRO received a negative score for all medicines, meaning that the PRO for the medications was worse when compared to the placebo. These scores were $-0.50 \pm 0.55$ for Vildagliptin, $-0.33 \pm 0.52$ for Alogliptin and $-0.17 \pm 0.41$ for Sitagliptin, Linagliptin and Saxagliptin.

In terms of cost, direct costs for Saxagliptin, Alogliptin and Sitagliptin which were purchased by our hospital were presented at the hospital price. Linagliptin and Vildagliptin were not located on the hospital purchased list and the mean price used in Chinese nine provinces was calculated. The costs of intervention received a negative score indicating that medicine cost was higher than placebo. The scores were $-1.83 \pm$ 1.33 for Saxagliptin and Aalogliptin, $-1.50 \pm 0.84$ for Sitagliptin, $-1.33 \pm 0.82$ for Linagliptin and $-1.17 \pm 0.98$ for Vildagliptin. There were no data available for other medical costs and thus the committee did not assign scores to these. Data was analyzed that compared DPP-4 inhibitors along with metformin as well as placebo along with metformin[17]. The committee believed that the medication showed greater nonmedical costs with higher variability. These scores were $-1.83 \pm 1.47$ for Saxagliptin, $-1.67 \pm 1.51$ for Alogliptin, $-1.50 \pm 1.05$ for Linagliptin, $-1.33 \pm 1.03$ for Sitagliptin and $-1.33 \pm 1.21$ for Vildagliptin.

\section{Estimated Value for five DPP-4 inhibitors}

The overall estimated value that integrated the weights and scores for five DPP-4 inhibitors all showed that the medications had higher added value compared to the placebo (Fig. 3). The overall value orders for the medications were 0.45 for Sitagliptin, 0.44 for Linagliptin, 0.43 for Vildagliptin, 0.42 for Alogliptin and 0.40 for Saxagliptin.

\section{Discussion}

The aim of this study was to assess the value of five DPP-4 inhibitors to aid the hospital in making decisions regarding drug optimization using the EVIDEM and MCDA guidelines. The MCDA has been used in China for the decision-making framework for medical insurance reimbursement and medical device assessment $[18,19]$. To our knowledge, this is the first time that MCDA and EVIDEM frameworks were used in hospital HTAs in China.

The hospital HTA group gave a value order for decision makers on the hospital PMC. After the assessment, Sitagliptin showed the highest value (mean of 0.45 ) and Saxagliptin received the lowest score (mean of 0.40). There was no major differences in the value between the two drugs. Interestingly, even though comparative data in the net-meta analysis showed no significant differences between the drugs, differences still existed[20-24]. Since the weights and scores generated by the committee inevitably revolve around their own perceptions, experience, training and value judgments, it is critical to have a multidisciplinary vision.

An HTA group in a hospital in Beijing evaluated DPP-4 inhibitors using a systematic review that qualitatively described effectiveness, safety and economics[25-27]. Different from this study, we gave a quantitative score for drug value using MCDA and included more comprehensive considerations for the 
value of medicines using the EVIDEM framework. All of these provided more specific suggestions for decision makers.

Even though this study provides guidance for decision makers, there are still some limitations. First, DPP-4 inhibitors were used in more treatment programs that are in clinical trials such as a DPP-4 inhibitor versus placebo/metformin/other anti-diabetes drugs or a DPP-4 inhibitor/anti-diabetes drugs versus other antidiabetes drugs[28, 29]. Based on time limitation and feasibility, we chose the placebo as the comparator. By only comparing to placebo, there is no consideration about the influence of other comparators. Secondly, in the terms of costs, sufficient available data in China was not obtainable since there was little research on Pharmacoeconomics from a Chinese social perspective, especially for the cost of drugs. Due to this, the panelist did not give a score for other-medical costs. Next, although the EVIDEM contained 13 widely identified criteria, other relevant criteria might have been excluded. Lastly, members of the committee and drug administration worked at our hospital making representation limited to a single hospital. Thus, the estimated value of the MCDA needs additional interpretations. The MCDA used for complementing pharmacoeconomic analyses which lack the broader perspective, meanwhile it also the discussion basis of ordering health technology $[30,31]$.

\section{Conclusion}

This pilot study assessed the value of five DPP-4 inhibitors to help the hospital in making decisions regarding drug optimization using MCDA. The overall value orders included Sitagliptin (0.45), Linagliptin (0.44), Vildagliptin (0.43), Alogliptin (0.42) and Saxagliptin (0.40). This was the first time that the MCDA and EVIDEM frameworks were used in a hospital HTA in China. This methodology aids in making the decision making process in hospitals more systematic and transparent. In the future, it was desired that MCDA was developing for more use, so that the decision-making of economic evaluation could have great transparency, efficiency, and consistency.

\section{Abbreviations}

PMC

Pharmacy Management Committee

EVIDEM

Evidence and Value:Impact on Decision Making

MCDA

Multi-Criteria Decision Analysis

HB-HTA

Hospital-Based Health Technology Assessment

HTA

Health Technology Assessment

\section{Declarations}




\section{Ethics approval and consent to participate}

This study was based on a literature review and MCDA; This study did not require approval by an Institutional Research Ethics Board.

\section{Consent for publication}

Not applicable.

\section{Availability of data and materials}

The datasets used and/or analyzed during the current study are available from the corresponding author on reasonable request.

\section{Competing interests}

Authors have no competing interests to declare.

\section{Funding}

This work was sponsored by Lanzhou Science and Technology Development Guiding Plan Project (No.2019-ZD-104), National Key R\&D Program of China (No. 2018YFC1311502), and National Natural Science Foundation of China (No. 81760144). The funders had no role in the study design, data collection and analysis, decision to publish, or manuscript preparation.

\section{Authors' contributions}

LMT and BYS was involved in the design of the study. MM and BG collected the data. YB wrote the first draft of the manuscript, which was critically revised by YB, BG, MM, BG, YY, CCD, BYS, and LMT.

\section{Acknowledgements}

Not applicable

\section{References}

1. Zeng Y, Yang M, Luo Y. Standardize the admission procedure of new drugs and optimize the variety of drugs in the hospital. Journal of Pediatric Pharmacy. 2004(05):40-41.

2. The basic establishment of the hospital drug administration system in China. Medicine and Society 2018, 31(07):13.

3. Angelis A, Lange A, Kanavos P. Using health technology assessment to assess the value of new medicines: results of a systematic review and expert consultation across eight European countries. Eur J Health Econ. 2018;19(1):123-52.

4. Schlander M. The use of cost-effectiveness by the National Institute for Health and Clinical Excellence (NICE): no(t yet an) exemplar of a deliberative process. J Med Ethics. 2008;34(7):534-9. 
5. Keeney RLRH: Decisions with Multiple Objectives: Preferences and Value Trade-Offs.. Cambridge University Press, Cambridge, UK, 1993.

6. Dolan JG. Medical Decision Making Using the Analytic Hierarchy Process:Choice of Initial Antimicrobial Therapy for Acute Pyelonephritis. Med Decis Making. 1989;9(1):51-6.

7. van Til JA, Renzenbrink GJ, Dolan JG, ljzerman MJ. The Use of the Analytic Hierarchy Process to Aid Decision Making in Acquired Equinovarus Deformity. Arch Phys Med Rehabil. 2008;89(3):457-62.

8. Muhlbacher AC, Kaczynski A. Making Good Decisions in Healthcare with Multi-Criteria Decision Analysis: The Use, Current Research and Future Development of MCDA. Appl Health Econ Health Policy. 2016;14(1):29-40.

9. EVIDEM Collaboration.. Decision criteria-conceptual back- ground, definitions, and instructions. https://wwwevidemorg/.

10. Goetghebeur MM, Wagner M, Khoury H, Levitt RJ, Erickson LJ, Rindress D. Evidence and Value: Impact on DEcisionMaking-the EVIDEM framework and potential applications. BMC Health Serv Res. 2008;8:270.

11. longzhao C. Inventory of DPP-4 inhibitors listed in China. https://medsinacom/article_detail_103_2_47380html 2018.

12. Thokala P, Devlin N, Marsh K, Baltussen R, Boysen M, Kalo Z, Longrenn T, Mussen F, Peacock S, Watkins J, et al. Multiple Criteria Decision Analysis for Health Care Decision Making-An Introduction: Report 1 of the ISPOR MCDA Emerging Good Practices Task Force. Value Health. 2016;19(1):1-13.

13. Marsh K, M IJ, Thokala P, Baltussen R, Boysen M, Kalo Z, Lonngren T, Mussen F, Peacock S, Watkins J, et al. Multiple Criteria Decision Analysis for Health Care Decision Making-Emerging Good Practices: Report 2 of the ISPOR MCDA Emerging Good Practices Task Force. Value Health. 2016;19(2):125-37.

14. Federation. ID: IDF Diabetes Atlas, 9th edn. https://diabetesatlasorg/en/ 2019.

15. Zhang Qi L, Yang T. Limin, et al: Epidemiology of diabetes in adults in Gansu province of China. Chin J Diabetes. 2019;27(01):3-6.

16. Whiting P, Savović J, Higgins JPT, Caldwell DM, Reeves BC, Shea B, Davies P, Kleijnen J, Churchill R. ROBIS: A new tool to assess risk of bias in systematic reviews was developed. Journal of Clinical Epidemiology 2016, 69.

17. Gao W: Short term efficacy of DPP-4inhibitors combined with metformin in the treatment of T2DM and its long term pharmacoeconmics evaluation. Shangdong University; 2016.

18. GJ,Chen Xiaowei, Yu Xiaolan, et al: Study on the evidence-based decision-making framework for reimbursement technologies in view of EVIDEM. Chinese Journal of Health Policy 2018, 11(04):50-54.

19. Wen Z: Evaluation Model of Medical Consumables Admission Based on EVIDEM and Its Application. Presented at the 2013 Proceedings of the 14th Academic Annual Meeting of the Chinese Medical Association Medical Engineering Branch Wuhan; 2013: 64-64.

20. Cai X, Gao X, Yang W, Chen Y, Zhou L, Zhang S, Han X, Ji L. DPP-4 Inhibitor Treatment in Chinese Type 2 Diabetes Patients: A Meta-Analysis. Diabetes Technol Ther. 2016;18(12):784-93. 
21. Craddy P, Palin HJ, Johnson KI. Comparative effectiveness of dipeptidylpeptidase-4 inhibitors in type 2 diabetes: a systematic review and mixed treatment comparison. Diabetes Ther. 2014;5(1):1-41.

22. Ling J, Cheng P, Ge L, Zhang DH, Shi AC, Tian JH, Chen YJ, Li XX, Zhang JY, Yang KH. The efficacy and safety of dipeptidyl peptidase-4 inhibitors for type 2 diabetes: a Bayesian network meta-analysis of 58 randomized controlled trials. Acta Diabetol. 2019;56(3):249-72.

23. Goossen K, Graber S. Longer term safety of dipeptidyl peptidase-4 inhibitors in patients with type 2 diabetes mellitus: systematic review and meta-analysis. Diabetes Obes Metab. 2012;14(12):1061-72.

24. Reaney M, Elash CA, Litcher-Kelly L. Patient Reported Outcomes (PROs) used in recent Phase 3 trials for Type 2 Diabetes: A review of concepts assessed by these PROs and factors to consider when choosing a PRO for future trials. Diabetes Res Clin Pract. 2016;116:54-67.

25. Men Peng,Gu Yunchun. Suodi Z. A rapid assessment of the effectiveness and safety of ritagliptin in the treatment of type 2 diabetes and its economic analysis in China. Chin J Pharmacoepidemiol 2017, 26(06):375-81.

26. Men P. Gu Yunchun, Zhai Suodi: Vildagliptin in the Treatment of Type 2 Diabetes Mellitus: Health Technology Assessment. Chin J Pharmacoepidemiol. 2016;25(09):533-40.

27. Peng M, Junwen Z. Tang Hhuilin, et al: Pharmacoeconomic Systematic Review of Saxagliptin for Type 2 Diabetes. Chinese Journal of Pharmacy. 2016;51(12):1044-8.

28. Gao W, Dong J, Liu J, Li Y, Liu F, Yang L, Zhou X, Liao L. Efficacy and safety of initial combination of DPP-IV inhibitors and metformin versus metformin monotherapy in type 2 diabetes: a systematic review of randomized controlled trials. Diabetes Obesity \& Metabolism, 16(2):179-185.

29. Wu S, Chai S, Yang J, Cai T, Zhan S: Gastrointestinal Adverse Events of Dipeptidyl Peptidase 4 Inhibitors in Type 2 Diabetes: A Systematic Review and Network Meta-analysis. Clinical Therapeutics 2017, 39(9):1780.

30. Tony $M$, Wagner $M$, Khoury $H$, Rindress $D$, Goetghebeur MM. Bridging health technology assessment (HTA) with multicriteria decision analyses (MCDA): Field testing of the EVIDEM framework for coverage decisions by a public payer in Canada. Bmc Health Services Research. 2011;11(1):329.

31. Baltussen R, Youngkong S, Paolucci F, Niessen L. Multi-criteria decision analysis to prioritize health interventions: Capitalizing on first experiences. Health Policy, 96(3):0-264.

\section{Figures}




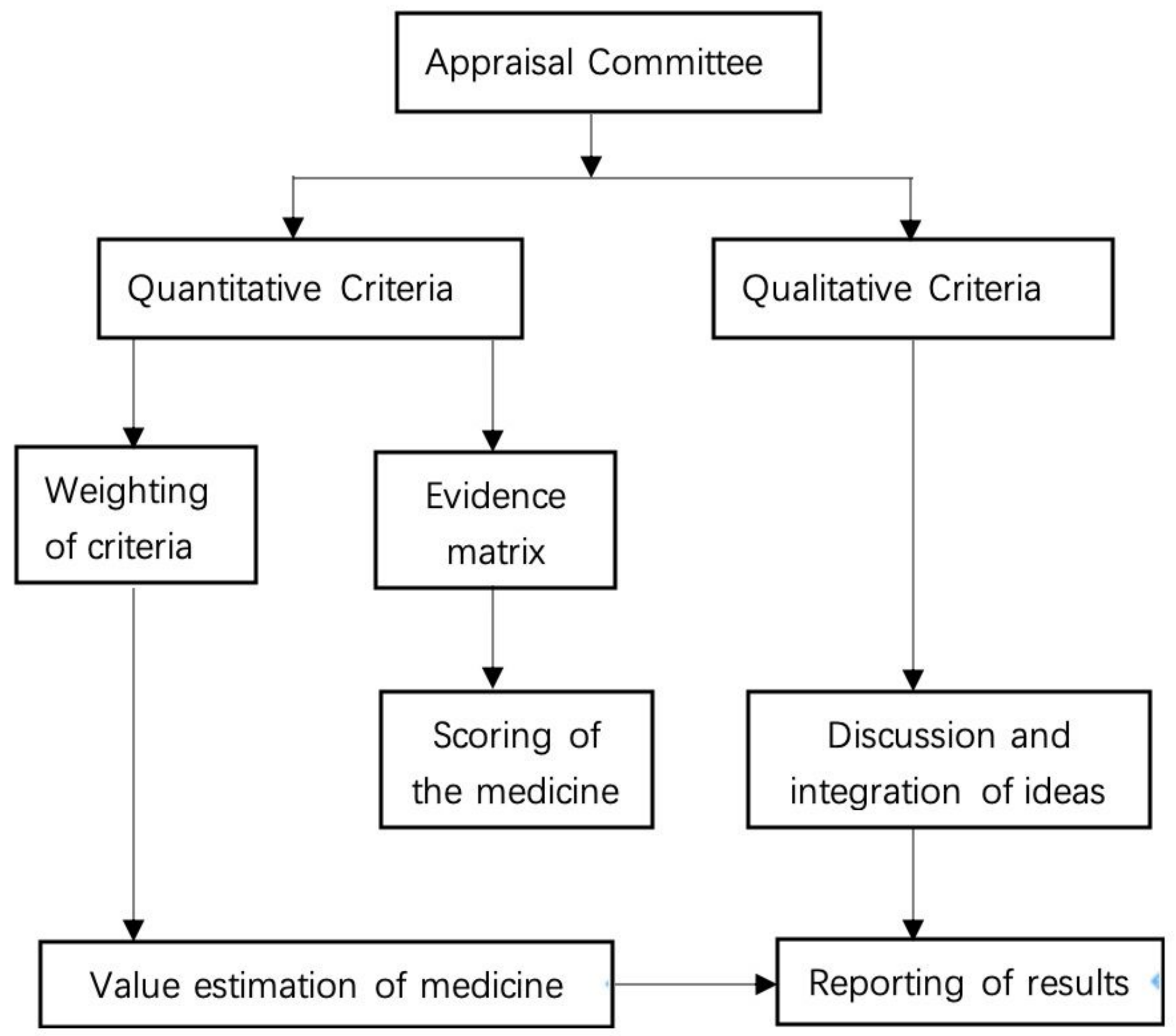

Figure 1

The estimating program 


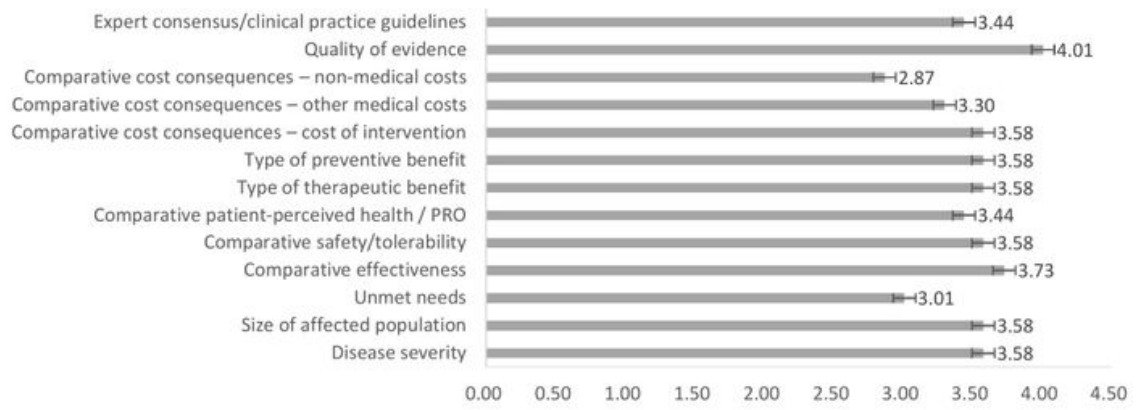

Figure 2

The mean weight for every criterion 


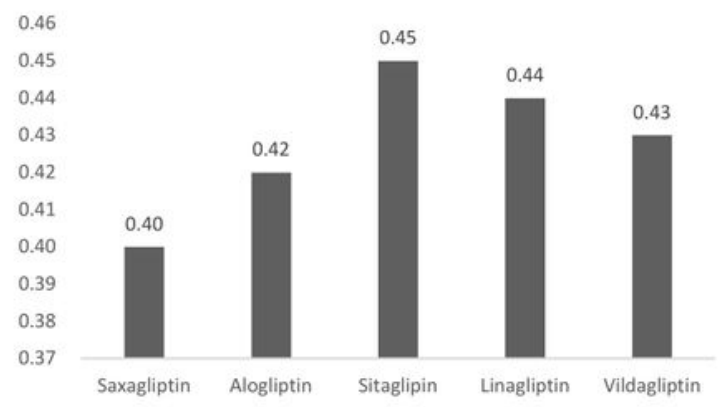

Figure 3

Estimated value for five DPP-4 inhibitors

Supplementary Files

This is a list of supplementary files associated with this preprint. Click to download. 
- Appendix1.docx

Page 15/15 